\title{
On the regularity criteria for the 3D magneto-micropolar fluids in terms of one directional derivative
}

\section{Zhaoyin Xiang* and Huizhi Yang}

\section{"Correspondence:}

zxiang@uestc.edu.cn

School of Mathematical Sciences, University of Electronic Science and

Technology of China, Chengdu, 611731, P.R. China

\begin{abstract}
In this paper, we establish two new regularity criteria for the 3D magneto-micropolar fluids in terms of one directional derivative of the velocity or of the pressure and the magnetic field.

MSC: 35Q35; 76W05; 35B65

Keywords: magneto-micropolar fluid equations; regularity criteria

\section{Introduction}

In this paper, we consider the Cauchy problem of the 3D incompressible magnetomicropolar fluid equations

$$
\left\{\begin{array}{l}
\partial_{t} u+u \cdot \nabla u-b \cdot \nabla b+\nabla\left(\pi+|b|^{2}\right)-\chi \nabla \times w=(\mu+\chi) \Delta u, \quad t \geq 0, x \in \mathbb{R}^{3}, \\
\partial_{t} w+u \cdot \nabla w-\kappa \nabla \operatorname{div} w+2 \chi w-\chi \nabla \times u=\gamma \Delta w, \quad t \geq 0, x \in \mathbb{R}^{3}, \\
\partial_{t} b+u \cdot \nabla b-b \cdot \nabla u=v \Delta b, \quad t \geq 0, x \in \mathbb{R}^{3}, \\
\operatorname{div} u=\operatorname{div} b=0, \quad t \geq 0, x \in \mathbb{R}^{3}, \\
u(x, 0)=u_{0}(x), \quad w(x, 0)=w_{0}(x), \quad b(x, 0)=b_{0}(x), \quad x \in \mathbb{R}^{3},
\end{array}\right.
$$
\end{abstract}

where $u$ is the fluid velocity, $w$ is the micro-rotational velocity, $b$ is the magnetic field and $\pi$ is the pressure. Equations (1.1) describe the motion of a micropolar fluid which is moving in the presence of a magnetic field (see [1]). The positive parameters $\mu, \chi, \gamma, \kappa$ and $\nu$ in (1.1) are associated with the properties of the materials: $\mu$ is the kinematic viscosity, $\chi$ is the vortex viscosity, $v$ and $\kappa$ are the spin viscosities and $\frac{1}{v}$ is the magnetic Reynolds number.

Recently, Yuan [2] investigated the local existence and uniqueness of the strong solutions to the magneto-micropolar fluid equations (1.1) (see also [3-6] for the bounded domain cases). Thus, the further problem at the center of the mathematical theory concerning equations (1.1) is whether or not it has a global in time smooth solution for any prescribed smooth initial data, which is still a challenging open problem. In the absence of a global well-posedness theory, the development of regularity criteria is of major importance for both theoretical and practical purposes. We would like to recall some related results in this direction.

Note that if the micro-rotation effects and the magnetic filed are not taken into account, i.e., $w=b=0$, equations (1.1) reduce to the classical Navier-Stokes equations. The global 
regularity issue has been thoroughly investigated for the 3D Navier-Stokes equations and many important regularity criteria have been established (see [7-16] and the references therein). In particular, the first well-known regularity criterion is due to Serrin [14]: if the Leray-Hopf weak solution $u$ of the 3D Navier-Stokes equations satisfies

$$
\int_{0}^{T}\|u(\cdot, t)\|_{L^{p}}^{q} \mathrm{~d} t<\infty \quad \text { with } \frac{2}{q}+\frac{3}{p}=1 \text { and } 3<p \leq \infty
$$

then $u$ is regular on $(0, T]$. Beirao da Veiga [8] and Penel and Pokorny [13] established another regularity criteria by replacing the above conditions with the following ones:

$$
\int_{0}^{T}\|\nabla u(\cdot, t)\|_{L^{p}}^{q} \mathrm{~d} t<\infty \quad \text { with } \frac{2}{q}+\frac{3}{p}=2 \text { and } \frac{3}{2}<p \leq \infty
$$

or

$$
\int_{0}^{T}\left\|\partial_{3} u(\cdot, t)\right\|_{L^{p}}^{q} \mathrm{~d} t<\infty \quad \text { with } \frac{2}{q}+\frac{3}{p} \leq \frac{3}{2} \text { and } 2 \leq p \leq \infty
$$

More recently, Cao and Titi [17] established a regularity criterion in terms of only one of the nine components of the gradient of a velocity field, that is, the solution $u$ is regular on $[0, T]$ if

$$
\int_{0}^{T}\left\|\partial_{k} u_{j}(\cdot, t)\right\|_{L^{p}}^{q} \mathrm{~d} t<\infty \quad \text { with } \frac{2}{q}+\frac{3}{p} \leq \frac{p+3}{2 p} \text { and } 3<p \leq \infty,
$$

where $k, j=1,2,3$ and $k \neq j$, or

$$
\int_{0}^{T}\left\|\partial_{j} u_{j}(\cdot, t)\right\|_{L^{p}}^{q} \mathrm{~d} t<\infty \quad \text { with } \frac{2}{q}+\frac{3}{p} \leq \frac{3(p+2)}{4 p} \text { and } 2<p \leq \infty
$$

This result on $\partial_{j} u_{j}$ is stronger than a similar result of Zhou and Pokorny [18] in the sense of allowing for much smaller values of $p$. These regularity criteria are of physical relevance since experimental measurements are usually obtained for quantities of the form $\partial_{k} u_{j}$. The regularity criterion by imposing the growth conditions on the pressure field are also examined by, for example, Berselli and Galdi [9], Chae and Lee [10] and Zhou [15, 16], i.e., if

$$
\int_{0}^{T}\|\pi(\cdot, t)\|_{L^{p}}^{q} \mathrm{~d} t<\infty \quad \text { with } \frac{2}{q}+\frac{3}{p}=2 \text { and } \frac{3}{2}<p \leq \infty,
$$

or

$$
\int_{0}^{T}\|\nabla \pi(\cdot, t)\|_{L^{p}}^{q} \mathrm{~d} t<\infty \quad \text { with } \frac{2}{q}+\frac{3}{p}=3 \text { and } 1<p \leq \infty
$$

then the solution $u$ is regular on $[0, T]$ (see also [14,17] for the Besov spaces cases). For the 3D Navier-Stokes equations with boundary conditions, Cao and Titi first introduced a regularity criterion in terms of only one component of the pressure gradient based on the breakthrough of the global regularity of the 3D primitive equations [19]. Recently, Cao 
and Titi [20] established a similar regularity criterion for the Cauchy problem of the 3D Navier-Stokes equations, that is, the solution $u$ is regular on $[0, T]$ if

$$
\int_{0}^{T}\left\|\partial_{3} \pi(\cdot, t)\right\|_{L^{p}}^{q} \mathrm{~d} t<\infty \quad \text { with } \frac{2}{q}+\frac{3}{p}<\frac{20}{7}, p>\frac{20}{16} \text { and } q \geq 1
$$

When the micro-rotation effects are neglected, i.e., $w=0$, equations (1.1) become the usual magnetohydrodynamic (MHD) equations. Some of the regularity criteria established for the Navier-Stokes equations can be extended to the 3D MHD equations by making assumptions on both $u$ and $b$ (see [21, 22]). Moreover, He and Xin [23, 24] showed that the velocity field $u$ plays a dominant role in the regularity issue and derived a criterion in terms of the velocity field $u$ alone (see also $[25,26]$ for the Besov spaces cases). Recently, Cao and $\mathrm{Wu}$ [27] further proved that if

$$
\int_{0}^{T}\left\|\partial_{3} u(\cdot, t)\right\|_{L^{p}}^{q} \mathrm{~d} t<\infty \quad \text { with } \frac{2}{q}+\frac{3}{p} \leq 1 \text { and } p \geq 3
$$

or

$$
\int_{0}^{T}\left\|\partial_{3} \pi(\cdot, t)\right\|_{L^{p}}^{q} \mathrm{~d} t<\infty \quad \text { with } \frac{2}{q}+\frac{3}{p} \leq \frac{7}{4} \text { and } p \geq \frac{12}{7}
$$

then $(u, b)$ is regular on [0,T]. More recently, Liu, Zhao and Cui [28] have adapted the method of [27] to establish a similar regularity criterion for the 3D nematic liquid crystal flow.

If we ignore the magnetic filed, i.e., $b=0$, equations (1.1) reduce to the micropolar fluid equations. The theory of micropolar fluid has attracted more and more scholars' attention in recent years. In particular, Dong, Jia and Chen [29] recently established a regularity criterion via the pressure field, which says that if

$$
\int_{0}^{T}\|\pi(\cdot, t)\|_{L^{p}}^{q} \mathrm{~d} t<\infty \quad \text { with } \frac{2}{q}+\frac{3}{p}=3 \text { and } 1<p \leq \infty,
$$

then $(u, w)$ is regular on $[0, T]$ (see also $[30,31]$ for the Lorentz spaces cases).

For the full magneto-micropolar fluid equations (1.1), Yuan [32] recently showed that the solution $(u, w, b)$ is regular on $(0, T]$ if

$$
\int_{0}^{T}\|u(\cdot, t)\|_{L^{p}}^{q} \mathrm{~d} t<\infty \quad \text { with } \frac{2}{q}+\frac{3}{p} \leq 1 \text { and } 3<p \leq \infty,
$$

or

$$
\int_{0}^{T}\|\nabla u(\cdot, t)\|_{L^{p}}^{q} \mathrm{~d} t<\infty \quad \text { with } \frac{2}{q}+\frac{3}{p} \leq 2 \text { and } \frac{3}{2}<p \leq \infty .
$$

For other regularity criteria of equations (1.1), we refer to Gala [33], Geng, Chen and Gala [34], Wang, Hu and Wang [35], Yuan [2] and Zhang, Yao and Wang [36].

In this paper, we establish two new regularity criteria for the $3 \mathrm{D}$ magneto-micropolar fluid equations (1.1) in terms of one directional derivative of the velocity $u$ or of the pressure $\pi$ and the magnetic field $b$ by adapting the method of [27]. Without loss of generality, we set the viscous coefficients $\mu+\chi=\gamma=\nu=\kappa=1$. 
We now state our main results as follows.

Theorem 1.1 Assume that $\left(u_{0}, w_{0}, b_{0}\right) \in H^{3}\left(\mathbb{R}^{3}\right)$ with $\operatorname{div} u_{0}=\operatorname{div} b_{0}=0$. Let $(u, w, b)$ be the corresponding local smooth solution to the magneto-micropolar fluid equations (1.1) on $[0, T)$ for some $T>0$. If the velocity $u$ satisfies

$$
\int_{0}^{T}\left\|\partial_{3} u(\cdot, t)\right\|_{L^{p}}^{q} \mathrm{~d} t:=M(T)<\infty \quad \text { with } \frac{2}{q}+\frac{3}{p} \leq 1 \text { and } p \geq 3,
$$

then $(u, w, b)$ can be extended beyond $T$.

Note that when $p=3, q=\infty$ and thus the corresponding assumption in (1.4) should be understood as $\operatorname{esssup}_{0 \leq t \leq T}\left\|\partial_{3} u(\cdot, t)\right\|_{L^{3}}:=M(T)<\infty$.

Remark 1.1 Theorem 1.1 improves the regularity criterion in [32] (see (1.3)) in the sense that it depends only on one directional derivative of the velocity $u$.

Theorem 1.2 Assume that $\left(u_{0}, w_{0}, b_{0}\right) \in H^{1}\left(\mathbb{R}^{3}\right) \cap L^{4}\left(\mathbb{R}^{3}\right)$ with $\operatorname{div} u_{0}=\operatorname{div} b_{0}=0$. Let $(u, w, b)$ be the corresponding local smooth solution to the magneto-micropolar fluid equations (1.1) on $[0, T)$ for some $T>0$. If the pressure $\pi$ and the magnetic field $b$ satisfy

$$
\int_{0}^{T}\left\|\partial_{3}\left(\pi(\cdot, t)+|b|^{2}(\cdot, t)\right)\right\|_{L^{p}}^{q} \mathrm{~d} t:=M(T)<\infty \quad \text { with } \frac{2}{q}+\frac{3}{p} \leq \frac{7}{4} \text { and } \frac{12}{7} \leq p \leq 4,
$$

then $(u, w, b)$ can be extended beyond $T$.

Remark 1.2 When $b=0$, we also obtain a new regularity criterion for the micropolar equations determined by one direction derivative of the pressure $\pi$ alone.

We shall prove our results in the next section. For simplicity, we denote by $\|\cdot\|_{p}$ the $L^{p}$ norm and by $(\cdot, \cdot)$ the $L^{2}$ inner product throughout the paper. The letter $C$ denotes an inessential constant which might vary from line to line, but does not depend on particular solutions or functions.

\section{Proof of the main results}

In this section, we give the proof of Theorem 1.1 and Theorem 1.2. The following lemma plays an important role in our arguments. Its proof can be found in [37] or [27].

Lemma 2.1 Let the parameters $r_{1}, r_{2}, r_{3}$ and $r$ satisfy

$$
1 \leq r_{1}, r_{2}, r_{3}, r<\infty \text { and } 1+\frac{3}{r}=\frac{1}{r_{1}}+\frac{1}{r_{2}}+\frac{1}{r_{3}}
$$

and suppose that $\partial_{i} \varphi \in L^{r_{i}}\left(\mathbb{R}^{3}\right)(i=1,2,3)$. Then there exists a constant $C=C\left(r_{1}, r_{2}, r_{3}\right)>0$ such that

$$
\|\varphi\|_{r} \leq C\left\|\partial_{1} \varphi\right\|_{r_{1}}^{\frac{1}{3}}\left\|\partial_{2} \varphi\right\|_{r_{2}}^{\frac{1}{3}}\left\|\partial_{3} \varphi\right\|_{r_{3}}^{\frac{1}{3}} .
$$


In particular, when $r_{1}=r_{2}=2$ and $r_{3}=p \in[1, \infty)$, there exists a constant $C=C(p)$ such that

$$
\|\varphi\|_{3 p} \leq C\left\|\partial_{1} \varphi\right\|_{2}^{\frac{1}{3}}\left\|\partial_{2} \varphi\right\|_{2}^{\frac{1}{3}}\left\|\partial_{3} \varphi\right\|_{p}^{\frac{1}{3}}
$$

for any $\varphi$ satisfying $\partial_{1} \varphi, \partial_{2} \varphi \in L^{2}\left(\mathbb{R}^{3}\right)$ and $\partial_{3} \varphi \in L^{p}\left(\mathbb{R}^{3}\right)$.

Proof of Theorem 1.1 Observe that for any $\left(u_{0}, w_{0}, b_{0}\right) \in H^{3}\left(\mathbb{R}^{3}\right)$ with $\operatorname{div} u_{0}=\operatorname{div} b_{0}=0$, there exists a unique local smooth solution to equations (1.1) (see [2]). Let $T_{0}$ be the maximum existence time. To prove Theorem 1.1, it is sufficient to show that the assumption (1.4) implies $T<T_{0}$. Indeed, we shall prove that under the condition (1.4), there exists a constant $C>0$ such that

$$
\limsup _{t \rightarrow T-}\left(\|\nabla u(t)\|_{2}^{2}+\|\nabla w(t)\|_{2}^{2}+\|\nabla b(t)\|_{2}^{2}\right) \leq C
$$

which implies that $T$ is not the maximum existence time and thus the solution $(u, w, b)$ can be extended beyond $T$ by the standard arguments of continuation of local solutions.

Firstly, we derive the energy inequality. For this purpose, we take the $L^{2}\left(\mathbb{R}^{3}\right)$ inner product of $u, w$ and $b$ with equations (1.1), respectively, sum the resulting equations and then integrate by parts to obtain

$$
\begin{aligned}
\frac{1}{2} \frac{\mathrm{d}}{\mathrm{d} t} & \left(\|u(t)\|_{2}^{2}+\|w(t)\|_{2}^{2}+\|b(t)\|_{2}^{2}\right) \\
& +\left(\|\nabla u(t)\|_{2}^{2}+\|\nabla w(t)\|_{2}^{2}+\|\nabla b(t)\|_{2}^{2}\right)+\|\operatorname{div} w(t)\|_{2}^{2}+2 \chi\|w(t)\|_{2}^{2} \\
= & \chi(u, \nabla \times w)+\chi(w, \nabla \times u) \\
\leq & \frac{1}{2}\left(\|\nabla u(t)\|_{2}^{2}+\|\nabla w(t)\|_{2}^{2}\right)+C\left(\|u(t)\|_{2}^{2}+\|w(t)\|_{2}^{2}\right)
\end{aligned}
$$

where we used $\operatorname{div} u=\operatorname{div} b=0$ in the first equality and Hölder's inequality in the last inequality. Thus,

$$
\begin{aligned}
& \frac{\mathrm{d}}{\mathrm{d} t}\left(\|u(t)\|_{2}^{2}+\|w(t)\|_{2}^{2}+\|b(t)\|_{2}^{2}\right) \\
& \quad+\left(\|\nabla u(t)\|_{2}^{2}+\|\nabla w(t)\|_{2}^{2}+\|\nabla b(t)\|_{2}^{2}\right)+\|\operatorname{div} w(t)\|_{2}^{2}+\chi\|w(t)\|_{2}^{2} \\
& \leq C\left(\|u(t)\|_{2}^{2}+\|w(t)\|_{2}^{2}\right) .
\end{aligned}
$$

It follows from Gronwall's inequality that

$$
\begin{aligned}
& \|u(t)\|_{2}^{2}+\|w(t)\|_{2}^{2}+\|b(t)\|_{2}^{2} \\
& \quad+\int_{0}^{t}\left(\|\nabla u(\tau)\|_{2}^{2}+\|\nabla w(\tau)\|_{2}^{2}+\|\nabla b(\tau)\|_{2}^{2}+\|\operatorname{div} w(\tau)\|_{2}^{2}+\chi\|w(\tau)\|_{2}^{2}\right) \mathrm{d} \tau \\
& \leq C e^{C t}\left(\left\|u_{0}\right\|_{2}^{2}+\left\|w_{0}\right\|_{2}^{2}++\left\|b_{0}\right\|_{2}^{2}\right) .
\end{aligned}
$$

Now we split the proof of the estimates (2.1) into two steps.

Step 1: Estimates for $\int_{0}^{T}\left(\left\|\nabla \partial_{3} u(\cdot, t)\right\|_{2}^{2}+\left\|\nabla \partial_{3} w(\cdot, t)\right\|_{2}^{2}+\left\|\nabla \partial_{3} b(\cdot, t)\right\|_{2}^{2}\right) \mathrm{d} t$. 
To this end, differentiating the first three equations in (1.1) with respect to $x_{3}$, taking the $L^{2}\left(\mathbb{R}^{3}\right)$ inner product of $\partial_{3} u, \partial_{3} w$ and $\partial_{3} b$ with the resulting equations, respectively, and then performing a space integration by parts, we get

$$
\begin{aligned}
& \frac{1}{2} \frac{\mathrm{d}}{\mathrm{d} t}\left\|\partial_{3} u(t)\right\|_{2}^{2}+\left\|\nabla \partial_{3} u\right\|_{2}^{2} \\
& \quad=-\left(\partial_{3} u, \partial_{3} u \cdot \nabla u\right)+\left(\partial_{3} u, \partial_{3} b \cdot \nabla b\right)+\left(\partial_{3} u, b \cdot \nabla \partial_{3} b\right)+\chi\left(\partial_{3} u, \nabla \times \partial_{3} w\right), \\
& \frac{1}{2} \frac{\mathrm{d}}{\mathrm{d} t}\left\|\partial_{3} w(t)\right\|_{2}^{2}+\left\|\nabla \partial_{3} w\right\|_{2}^{2}+\left\|\operatorname{div} \partial_{3} w\right\|_{2}^{2}+2 \chi\left\|\partial_{3} w\right\|_{2}^{2} \\
& \quad=-\left(\partial_{3} w, \partial_{3} u \cdot \nabla w\right)+\chi\left(\partial_{3} w, \nabla \times \partial_{3} u\right), \\
& \frac{1}{2} \frac{\mathrm{d}}{\mathrm{d} t}\left\|\partial_{3} b(t)\right\|_{2}^{2}+\left\|\nabla \partial_{3} b\right\|_{2}^{2}=-\left(\partial_{3} b, \partial_{3} u \cdot \nabla b\right)+\left(\partial_{3} b, \partial_{3} b \cdot \nabla u\right)+\left(\partial_{3} b, b \cdot \nabla \partial_{3} u\right),
\end{aligned}
$$

where we used the facts

$$
\left(\partial_{3} u, u \cdot \nabla \partial_{3} u\right)=\left(\partial_{3} u, \nabla \partial_{3}\left(\pi+|b|^{2}\right)\right)=\left(\partial_{3} w, u \cdot \nabla \partial_{3} w\right)=\left(\partial_{3} b, u \cdot \nabla \partial_{3} b\right)=0
$$

by $\operatorname{div} u=0$. Noticing that

$$
\left(\partial_{3} u, \nabla \times \partial_{3} w\right)=\left(\partial_{3} w, \nabla \times \partial_{3} u\right) \quad \text { and } \quad\left(\partial_{3} u, b \cdot \nabla \partial_{3} b\right)+\left(\partial_{3} b, b \cdot \nabla \partial_{3} u\right)=0
$$

by $\operatorname{div} b=0$, we can sum the above equations to obtain

$$
\begin{aligned}
\frac{1}{2} \frac{\mathrm{d}}{\mathrm{d} t} & \left(\left\|\partial_{3} u(t)\right\|_{2}^{2}+\left\|\partial_{3} w(t)\right\|_{2}^{2}+\left\|\partial_{3} b(t)\right\|_{2}^{2}\right) \\
& +\left(\left\|\nabla \partial_{3} u\right\|_{2}^{2}+\left\|\nabla \partial_{3} w\right\|_{2}^{2}+\left\|\nabla \partial_{3} b\right\|_{2}^{2}\right)+\left\|\operatorname{div} \partial_{3} w\right\|_{2}^{2}+2 \chi\left\|\partial_{3} w\right\|_{2}^{2} \\
= & -\left(\partial_{3} u, \partial_{3} u \cdot \nabla u\right)+\left(\partial_{3} u, \partial_{3} b \cdot \nabla b\right)-\left(\partial_{3} w, \partial_{3} u \cdot \nabla w\right)+2 \chi\left(\partial_{3} w, \nabla \times \partial_{3} u\right) \\
& \quad-\left(\partial_{3} b, \partial_{3} u \cdot \nabla b\right)+\left(\partial_{3} b, \partial_{3} b \cdot \nabla u\right) \\
:= & I_{1}+I_{2}+I_{3}+I_{4}+I_{5}+I_{6} .
\end{aligned}
$$

We now estimate the above terms one by one. To bound $I_{1}$, we first integrate by parts and then apply Hölder's inequality to obtain

$$
\left|I_{1}\right|=\left|\left(u, \partial_{3} u \cdot \nabla \partial_{3} u\right)\right| \leq\left\|\nabla \partial_{3} u\right\|_{2}\left\|\partial_{3} u\right\|_{\frac{6 p}{3 p-2}}\|u\|_{3 p}
$$

It follows from the Gagliardo-Nirenberg inequality that

$$
\left\|\partial_{3} u\right\|_{\frac{6 p}{3 p-2}} \leq C\left\|\nabla \partial_{3} u\right\|_{2}^{\frac{1}{p}}\left\|\partial_{3} u\right\|_{2}^{1-\frac{1}{p}}
$$

and from Lemma 2.1 that

$$
\|u\|_{3 p} \leq C\left\|\partial_{1} u\right\|_{2}^{\frac{1}{3}}\left\|\partial_{2} u\right\|_{2}^{\frac{1}{3}}\left\|\partial_{3} u\right\|_{p}^{\frac{1}{3}} \leq C\|\nabla u\|_{2}^{\frac{2}{3}}\left\|\partial_{3} u\right\|_{p}^{\frac{1}{3}} .
$$


Substituting these two estimates into (2.3) and then using Young's inequality, we see that for $p>3$

$$
\begin{aligned}
\left|I_{1}\right| & \leq C\left\|\nabla \partial_{3} u\right\|_{2}^{1+\frac{1}{p}}\left\|\partial_{3} u\right\|_{2}^{1-\frac{1}{p}}\|\nabla u\|_{2}^{\frac{2}{3}}\left\|\partial_{3} u\right\|_{p}^{\frac{1}{3}} \\
& \leq \frac{1}{4}\left\|\nabla \partial_{3} u\right\|_{2}^{2}+C\left\|\partial_{3} u\right\|_{2}^{2}\|\nabla u\|_{2}^{\frac{4 p}{3(p-1)}}\left\|\partial_{3} u\right\|_{p}^{\frac{2 p}{3(p-1)}} \\
& \leq \frac{1}{4}\left\|\nabla \partial_{3} u\right\|_{2}^{2}+C\left\|\partial_{3} u\right\|_{2}^{2}\left(\|\nabla u\|_{2}^{2}+\left\|\partial_{3} u\right\|_{p}^{\frac{2 p}{p-3}}\right)
\end{aligned}
$$

and that for $p=3$

$$
\begin{aligned}
\left|I_{1}\right| & \leq C\left\|\nabla \partial_{3} u\right\|_{2}^{\frac{4}{3}}\left\|\partial_{3} u\right\|_{2}^{\frac{2}{3}}\|\nabla u\|_{2}^{\frac{2}{3}}\left\|\partial_{3} u\right\|_{3}^{\frac{1}{3}} \\
& \leq \frac{1}{4}\left\|\nabla \partial_{3} u\right\|_{2}^{2}+C\left\|\partial_{3} u\right\|_{2}^{2}\left(\|\nabla u\|_{2}^{2}\left\|\partial_{3} u\right\|_{3}\right) .
\end{aligned}
$$

For $I_{2}$, by Hölder's inequality, the Gagliardo-Nirenberg inequality and Young's inequality, we have for $p>3$

$$
\begin{aligned}
\left|I_{2}\right| & \leq\|\nabla b\|_{2}\left\|\partial_{3} u\right\|_{p}\left\|\partial_{3} b\right\|_{\frac{2 p}{p-2}} \\
& \leq C\|\nabla b\|_{2}\left\|\partial_{3} u\right\|_{p}\left\|\partial_{3} b\right\|_{2}^{1-\frac{3}{p}}\left\|\nabla \partial_{3} b\right\|_{2}^{\frac{3}{p}} \\
& \leq \frac{1}{8}\left\|\nabla \partial_{3} b\right\|_{2}^{2}+C\|\nabla b\|_{2}^{\frac{2 p}{2 p-3}}\left\|\partial_{3} u\right\|_{p}^{\frac{2 p}{2 p-3}}\left\|\partial_{3} b\right\|_{2}^{\frac{2 p-6}{2 p-3}} \\
& \leq \frac{1}{8}\left\|\nabla \partial_{3} b\right\|_{2}^{2}+C\left(\|\nabla b\|_{2}^{2}+\left\|\partial_{3} u\right\|_{p}^{\frac{2 p}{p-3}}\right)\left\|\partial_{3} b\right\|_{2}^{\frac{2 p-6}{2 p-3}} \\
& \leq \frac{1}{8}\left\|\nabla \partial_{3} b\right\|_{2}^{2}+C\left(\|\nabla b\|_{2}^{2}+\left\|\partial_{3} u\right\|_{p}^{\frac{2 p}{p-3}}\right)\left(1+\left\|\partial_{3} b\right\|_{2}^{2}\right),
\end{aligned}
$$

and for $p=3$

$$
\begin{aligned}
\left|I_{2}\right| & \leq\|\nabla b\|_{2}\left\|\partial_{3} u\right\|_{3}\left\|\partial_{3} b\right\|_{6} \leq C\|\nabla b\|_{2}\left\|\partial_{3} u\right\|_{3}\left\|\nabla \partial_{3} b\right\|_{2} \\
& \leq \frac{1}{8}\left\|\nabla \partial_{3} b\right\|_{2}^{2}+C\|\nabla b\|_{2}^{2}\left\|\partial_{3} u\right\|_{3}^{2} .
\end{aligned}
$$

Applying similar procedure to $I_{3}$ and $I_{5}$, we have for $p<3$

$$
\left|I_{3}\right| \leq \frac{1}{2}\left\|\nabla \partial_{3} w\right\|_{2}^{2}+C\left(\|\nabla w\|_{2}^{2}+\left\|\partial_{3} u\right\|_{p}^{\frac{2 p}{p-3}}\right)\left(1+\left\|\partial_{3} w\right\|_{2}^{2}\right)
$$

and

$$
\left|I_{5}\right| \leq \frac{1}{8}\left\|\nabla \partial_{3} b\right\|_{2}^{2}+C\left(\|\nabla b\|_{2}^{2}+\left\|\partial_{3} u\right\|_{p}^{\frac{2 p}{p-3}}\right)\left(1+\left\|\partial_{3} b\right\|_{2}^{2}\right)
$$

and for $p=3$

$$
\left|I_{3}\right| \leq \frac{1}{8}\left\|\nabla \partial_{3} w\right\|_{2}^{2}+C\|\nabla w\|_{2}^{2}\left\|\partial_{3} u\right\|_{3}^{2}, \quad\left|I_{5}\right| \leq \frac{1}{8}\left\|\nabla \partial_{3} b\right\|_{2}^{2}+C\|\nabla b\|_{2}^{2}\left\|\partial_{3} u\right\|_{3}^{2} .
$$


For the term $I_{4}$, by using Hölder's inequality and Young's inequality, it can be bounded as follows:

$$
\left|I_{4}\right|=2 \chi\left(\partial_{3} w, \nabla \times \partial_{3} u\right) \leq \frac{1}{4}\left\|\nabla \partial_{3} u\right\|_{2}^{2}+C\left\|\partial_{3} w\right\|_{2}^{2} .
$$

Finally, we can follow the steps as in the bound of $I_{1}$ to estimate $I_{6}$. Precisely, by integrations by parts and Hölder's inequality, we have

$$
\left|I_{6}\right|=\left|\left(\partial_{3} b, \partial_{3} b \cdot \nabla u\right)\right|=\left|\left(u, \partial_{3} b \cdot \nabla \partial_{3} b\right)\right| \leq\left\|\nabla \partial_{3} b\right\|_{2}\left\|\partial_{3} b\right\|_{\frac{6 p}{3 p-2}}\|u\|_{3 p} .
$$

Then the Gagliardo-Nirenberg inequality, Lemma 2.1 and Young's inequality yield that for $p<3$

$$
\begin{aligned}
\left|I_{6}\right| & \leq C\left\|\nabla \partial_{3} b\right\|_{2}^{1+\frac{1}{p}}\left\|\partial_{3} b\right\|_{2}^{1-\frac{1}{p}}\|\nabla u\|_{2}^{\frac{2}{3}}\left\|\partial_{3} u\right\|_{p}^{\frac{1}{3}} \\
& \leq \frac{1}{4}\left\|\nabla \partial_{3} b\right\|_{2}^{2}+C\left\|\partial_{3} b\right\|_{2}^{2}\|\nabla u\|_{2}^{\frac{4 p}{3(p-1)}}\left\|\partial_{3} u\right\|_{p}^{\frac{2 p}{3(p-1)}} \\
& \leq \frac{1}{4}\left\|\nabla \partial_{3} b\right\|_{2}^{2}+C\left\|\partial_{3} b\right\|_{2}^{2}\left(\|\nabla u\|_{2}^{2}+\left\|\partial_{3} u\right\|_{p}^{\frac{2 p}{p-3}}\right),
\end{aligned}
$$

and for $p=3$

$$
\begin{aligned}
\left|I_{6}\right| & \leq C\left\|\nabla \partial_{3} b\right\|_{2}^{\frac{4}{3}}\left\|\partial_{3} b\right\|_{2}^{\frac{2}{3}}\|\nabla u\|_{2}^{\frac{2}{3}}\left\|\partial_{3} u\right\|_{3}^{\frac{1}{3}} \\
& \leq \frac{1}{4}\left\|\nabla \partial_{3} b\right\|_{2}^{2}+C\left\|\partial_{3} b\right\|_{2}^{2}\left(\|\nabla u\|_{2}^{2}\left\|\partial_{3} u\right\|_{3}\right) .
\end{aligned}
$$

Combining the estimates (2.4)-(2.12), we see that for $p>3$

$$
\begin{aligned}
& \frac{\mathrm{d}}{\mathrm{d} t}\left(\left\|\partial_{3} u(t)\right\|_{2}^{2}+\left\|\partial_{3} w(t)\right\|_{2}^{2}+\left\|\partial_{3} b(t)\right\|_{2}^{2}\right) \\
& \quad+\left(\left\|\nabla \partial_{3} u\right\|_{2}^{2}+\left\|\nabla \partial_{3} w\right\|_{2}^{2}+\left\|\nabla \partial_{3} b\right\|_{2}^{2}\right)+\left\|\operatorname{div} \partial_{3} w\right\|_{2}^{2}+\chi\left\|\partial_{3} w\right\|_{2}^{2} \\
& \quad \leq C\left(1+\left\|\partial_{3} u\right\|_{2}^{2}+\left\|\partial_{3} w\right\|_{2}^{2}+\left\|\partial_{3} b\right\|_{2}^{2}\right)\left(1+\|\nabla u\|_{2}^{2}+\|\nabla w\|_{2}^{2}+\|\nabla b\|_{2}^{2}+\left\|\partial_{3} u\right\|_{p}^{\frac{2 p}{p-3}}\right),
\end{aligned}
$$

and that for $p=3$

$$
\begin{aligned}
& \frac{\mathrm{d}}{\mathrm{d} t}\left(\left\|\partial_{3} u(t)\right\|_{2}^{2}+\left\|\partial_{3} w(t)\right\|_{2}^{2}+\left\|\partial_{3} b(t)\right\|_{2}^{2}\right) \\
& \quad+\left(\left\|\nabla \partial_{3} u\right\|_{2}^{2}+\left\|\nabla \partial_{3} w\right\|_{2}^{2}+\left\|\nabla \partial_{3} b\right\|_{2}^{2}\right)+\left\|\operatorname{div} \partial_{3} w\right\|_{2}^{2}+\chi\left\|\partial_{3} w\right\|_{2}^{2} \\
& \quad \leq C\left(1+\left\|\partial_{3} u\right\|_{2}^{2}+\left\|\partial_{3} w\right\|_{2}^{2}+\left\|\partial_{3} b\right\|_{2}^{2}\right)\left(1+\|\nabla u\|_{2}^{2}+\|\nabla w\|_{2}^{2}+\|\nabla b\|_{2}^{2}\right)\left(1+\left\|\partial_{3} u\right\|_{3}^{2}\right) .
\end{aligned}
$$

Thus, Gronwall's inequality together with the energy inequality (2.2) and the assumption (1.4) implies that for $p>3$

$$
\begin{aligned}
& \left(\left\|\partial_{3} u(t)\right\|_{2}^{2}+\left\|\partial_{3} w(t)\right\|_{2}^{2}+\left\|\partial_{3} b(t)\right\|_{2}^{2}\right) \\
& \quad \leq\left(1+\left\|\partial_{3} u_{0}\right\|_{2}^{2}+\left\|\partial_{3} w_{0}\right\|_{2}^{2}+\left\|\partial_{3} b_{0}\right\|_{2}^{2}\right) e^{C \int_{0}^{t}\left(1+\|\nabla u(\tau)\|_{2}^{2}+\|\nabla w(\tau)\|_{2}^{2}+\|\nabla b(\tau)\|_{2}^{2}+\left\|\partial_{3} u(\tau)\right\|_{p}^{\frac{2 p}{p-3}}\right.} \mathrm{d} \tau
\end{aligned}
$$




$$
\begin{aligned}
& \leq\left(1+\left\|\partial_{3} u_{0}\right\|_{2}^{2}+\left\|\partial_{3} w_{0}\right\|_{2}^{2}+\left\|\partial_{3} b_{0}\right\|_{2}^{2}\right) e^{C e^{C t}\left(1+\left\|u_{0}\right\|_{2}^{2}+\left\|w_{0}\right\|_{2}^{2}+\left\|b_{0}\right\|_{2}^{2}\right)+C M(t)^{q^{*} / q_{t}} t^{1-q^{\prime \prime} / q}} \\
& :=G_{p}(M(t))<\infty \quad(t \leq T)
\end{aligned}
$$

with $q^{*}=2 p /(p-3)$, and

$$
\begin{aligned}
& \left(\left\|\partial_{3} u(t)\right\|_{2}^{2}+\left\|\partial_{3} w(t)\right\|_{2}^{2}+\left\|\partial_{3} b(t)\right\|_{2}^{2}\right) \\
& \quad \leq\left(1+\left\|\partial_{3} u_{0}\right\|_{2}^{2}+\left\|\partial_{3} w_{0}\right\|_{2}^{2}+\left\|\partial_{3} b_{0}\right\|_{2}^{2}\right) e^{C \int_{0}^{t}\left(1+\|\nabla u(\tau)\|_{2}^{2}+\|\nabla w(\tau)\|_{2}^{2}+\|\nabla b(\tau)\|_{2}^{2}\right)\left(1+\left\|\partial_{3} u(\tau)\right\|_{3}^{2}\right) \mathrm{d} \tau} \\
& \quad \leq\left(1+\left\|\partial_{3} u_{0}\right\|_{2}^{2}+\left\|\partial_{3} w_{0}\right\|_{2}^{2}+\left\|\partial_{3} b_{0}\right\|_{2}^{2}\right) e^{C e^{C t}\left(1+\left\|u_{0}\right\|_{2}^{2}+\left\|w_{0}\right\|_{2}^{2}+\left\|b_{0}\right\|_{2}^{2}\right)\left(t+M(t)^{2}\right)} \\
& \quad:=G_{3}(M(t))<\infty \quad(t \leq T) .
\end{aligned}
$$

Then

$$
\begin{aligned}
& \int_{0}^{T}\left(\left\|\nabla \partial_{3} u(\tau)\right\|_{2}^{2}+\left\|\nabla \partial_{3} w(\tau)\right\|_{2}^{2}+\left\|\nabla \partial_{3} b(\tau)\right\|_{2}^{2}+\left\|\operatorname{div} \partial_{3} w(\tau)\right\|_{2}^{2}+\chi\left\|\partial_{3} w(\tau)\right\|_{2}^{2}\right) \mathrm{d} \tau \\
& \quad \leq \tilde{G}(M(t))<\infty
\end{aligned}
$$

which is the desired estimates.

Step 2: Estimates for $\left(\|\nabla u(t)\|_{2}+\|\nabla w(t)\|_{2}+\|\nabla b(t)\|_{2}\right)$.

For this purpose, taking the $L^{2}\left(\mathbb{R}^{3}\right)$ inner product of $\Delta u, \Delta w$ and $\Delta b$ with the first three equations in (1.1), respectively, and then performing a space integration by parts, we have

$$
\begin{aligned}
& \frac{1}{2} \frac{\mathrm{d}}{\mathrm{d} t}\|\nabla u(t)\|_{2}^{2}+\|\Delta u\|_{2}^{2}=(\Delta u, u \cdot \nabla u)-(\Delta u, b \cdot \nabla b)-\chi(\Delta u, \nabla \times w), \\
& \frac{1}{2} \frac{\mathrm{d}}{\mathrm{d} t}\|\nabla w(t)\|_{2}^{2}+\|\Delta w\|_{2}^{2}+\|\nabla \operatorname{div} w\|_{2}^{2}+2 \chi\|\nabla w\|_{2}^{2}=(\Delta w, u \cdot \nabla w)-\chi(\Delta w, \nabla \times u), \\
& \frac{1}{2} \frac{\mathrm{d}}{\mathrm{d} t}\|\nabla b(t)\|_{2}^{2}+\|\Delta b\|_{2}^{2}=(\Delta b, u \cdot \nabla b)-(\Delta b, b \cdot \nabla u) .
\end{aligned}
$$

Noticing $(\Delta u, \nabla \times w)=(\Delta w, \nabla \times u)$, we sum the above equations and integrate by parts to obtain

$$
\begin{aligned}
\frac{1}{2} \frac{\mathrm{d}}{\mathrm{d} t}\left(\|\nabla u(t)\|_{2}^{2}+\|\nabla w(t)\|_{2}^{2}+\|\nabla b(t)\|_{2}^{2}\right) & \\
& +\left(\|\Delta u\|_{2}^{2}+\|\Delta w\|_{2}^{2}+\|\Delta b\|_{2}^{2}\right)+\|\nabla \operatorname{div} w\|_{2}^{2}+2 \chi\|\nabla w\|_{2}^{2} \\
= & (\Delta u, u \cdot \nabla u)-(\Delta u, b \cdot \nabla b)+(\Delta b, u \cdot \nabla b) \\
& \quad-(\Delta b, b \cdot \nabla u)+(\Delta w, u \cdot \nabla w)-2 \chi(\Delta w, \nabla \times u) \\
= & \left(-\sum_{k=1}^{3}\left(\partial_{k} u, \partial_{k} u \cdot \nabla u\right)+\sum_{k=1}^{3}\left(\partial_{k} u, \partial_{k} b \cdot \nabla b\right)+\sum_{k=1}^{3}\left(\partial_{k} b, \partial_{k} b \cdot \nabla u\right)\right. \\
& \left.\quad-\sum_{k=1}^{3}\left(\partial_{k} b, \partial_{k} u \cdot \nabla b\right)-\sum_{k=1}^{3}\left(\partial_{k} w, \partial_{k} u \cdot \nabla w\right)\right)-2 \chi(\Delta w, \nabla \times u) \\
\leq & \left(\|\nabla u\|_{3}^{3}+3\|\nabla u\|_{3}\|\nabla b\|_{3}^{2}+\|\nabla u\|_{3}\|\nabla w\|_{3}^{2}\right)+\frac{1}{4}\|\Delta w\|_{2}^{2}+C\|\nabla u\|_{2}^{2} .
\end{aligned}
$$


By using the interpolation inequality and taking $p=2$ in Lemma 2.1, we have

$$
\|\nabla u\|_{3}^{3} \leq C\left(\|\nabla u\|_{2}^{\frac{1}{2}}\|\nabla u\|_{6}^{\frac{1}{2}}\right)^{3} \leq C\left(\|\nabla u\|_{2}^{\frac{1}{2}}\left\|\nabla_{h} \nabla u\right\|_{2}^{\frac{1}{3}}\left\|\partial_{3} \nabla u\right\|_{2}^{\frac{1}{6}}\right)^{3},
$$

where $\nabla_{h}=\left(\partial_{1}, \partial_{2}\right)$. Then Young's inequality yields

$$
\begin{aligned}
\|\nabla u\|_{3}^{3} & \leq \frac{1}{4}\left\|\nabla_{h} \nabla u\right\|_{2}^{2}+C\|\nabla u\|_{2}^{3}\left\|\partial_{3} \nabla u\right\|_{2} \\
& \leq \frac{1}{4}\left\|\nabla_{h} \nabla u\right\|_{2}^{2}+C\left(\|\nabla u\|_{2}^{2}+\left\|\partial_{3} \nabla u\right\|_{2}^{2}\right)\|\nabla u\|_{2}^{2}
\end{aligned}
$$

Similarly,

$$
\begin{aligned}
3 \| \nabla & u\left\|_{3}\right\| \nabla b\left\|_{3}^{2}+\right\| \nabla u\left\|_{3}\right\| \nabla w \|_{3}^{2} \\
\leq & 4\|\nabla u\|_{3}^{3}+3\|\nabla b\|_{3}^{3}+\|\nabla w\|_{3}^{3} \\
\leq & \frac{1}{4}\left(\left\|\nabla_{h} \nabla u\right\|_{2}^{2}+\left\|\nabla_{h} \nabla b\right\|_{2}^{2}+\left\|\nabla_{h} \nabla w\right\|_{2}^{2}\right) \\
& +C\left(\|\nabla u\|_{2}^{2}+\left\|\partial_{3} \nabla u\right\|_{2}^{2}\right)\|\nabla u\|_{2}^{2}+C\left(\|\nabla b\|_{2}^{2}+\left\|\partial_{3} \nabla b\right\|_{2}^{2}\right)\|\nabla b\|_{2}^{2} \\
& +C\left(\|\nabla w\|_{2}^{2}+\left\|\partial_{3} \nabla w\right\|_{2}^{2}\right)\|\nabla w\|_{2}^{2} .
\end{aligned}
$$

Substituting the above two estimates into (2.15), we have

$$
\begin{aligned}
\frac{\mathrm{d}}{\mathrm{d} t}\left(\|\nabla u(t)\|_{2}^{2}+\|\nabla w(t)\|_{2}^{2}+\|\nabla b(t)\|_{2}^{2}\right) & \\
& +\left(\|\Delta u\|_{2}^{2}+\|\Delta w\|_{2}^{2}+\|\Delta b\|_{2}^{2}\right)+\|\nabla \operatorname{div} w\|_{2}^{2}+\chi\|\nabla w\|_{2}^{2} \\
\leq & C\left(1+\|\nabla u\|_{2}^{2}+\left\|\partial_{3} \nabla u\right\|_{2}^{2}+\|\nabla w\|_{2}^{2}+\left\|\partial_{3} \nabla w\right\|_{2}^{2}+\|\nabla b\|_{2}^{2}+\left\|\partial_{3} \nabla b\right\|_{2}^{2}\right) \\
& \times\left(\|\nabla u\|_{2}^{2}+\|\nabla w\|_{2}^{2}+\|\nabla b\|_{2}^{2}\right) .
\end{aligned}
$$

By using Gronwall's inequality, the energy inequality (2.2) and the estimate (2.14), we conclude that

$$
\begin{aligned}
& \left(\|\nabla u(t)\|_{2}^{2}+\|\nabla w(t)\|_{2}^{2}+\|\nabla b(t)\|_{2}^{2}\right) \\
& \quad+\int_{0}^{t}\left(\|\Delta u(\tau)\|_{2}^{2}+\|\Delta w(\tau)\|_{2}^{2}+\|\Delta b(\tau)\|_{2}^{2}+\|\nabla \operatorname{div} w(\tau)\|_{2}^{2}+\chi\|\nabla w(\tau)\|_{2}^{2}\right) \mathrm{d} \tau \\
& \leq\left(\left\|\nabla u_{0}\right\|_{2}^{2}+\left\|\nabla w_{0}\right\|_{2}^{2}+\left\|\nabla b_{0}\right\|_{2}^{2}\right) \\
& \quad \times e^{C \int_{0}^{t}\left(1+\|\nabla u(\tau)\|_{2}^{2}+\left\|\partial_{3} \nabla u(\tau)\right\|_{2}^{2}+\|\nabla w(\tau)\|_{2}^{2}+\left\|\partial_{3} \nabla w(\tau)\right\|_{2}^{2}+\|\nabla b(\tau)\|_{2}^{2}+\left\|\partial_{3} \nabla b(\tau)\right\|_{2}^{2}\right) \mathrm{d} \tau} \\
& \leq C \tilde{G}(M(t))<\infty
\end{aligned}
$$

for any $t \leq T$, which implies that the desired estimates (2.1) hold and thus the solution $(u, w, b)$ can be extended beyond $T$.

Now we turn our attention to proving Theorem 1.2. We will first transform equations (1.1) into a symmetric form. 
Proof of Theorem 1.2 Following from Serrin type criteria (1.2) with $p=4$ and $q=\infty$ on the 3D magneto-micropolar fluid equations (1.1), it is sufficient to prove that

$$
\lim _{t \rightarrow T-}\left(\|u(t)\|_{4}+\|w(t)\|_{4}+\|b(t)\|_{4}\right)<\infty
$$

To do this, we set

$$
v^{+}=u+b, \quad v^{-}=u-b,
$$

and then equations (1.1) are converted to the following symmetric form:

$$
\left\{\begin{array}{l}
\partial_{t} v^{+}+v^{-} \cdot \nabla v^{+}+\nabla\left(\pi+|b|^{2}\right)-\chi \nabla \times w=\Delta v^{+}, \quad t \geq 0, x \in \mathbb{R}^{3}, \\
\partial_{t} w+\frac{1}{2}\left(v^{+}+v^{-}\right) \cdot \nabla w-\nabla \operatorname{div} w+2 \chi w \\
\quad-\frac{\chi}{2} \nabla \times\left(v^{+}+v^{-}\right)=\Delta w, \quad t \geq 0, x \in \mathbb{R}^{3}, \\
\partial_{t} v^{-}+v^{+} \cdot \nabla v^{-}+\nabla\left(\pi+|b|^{2}\right)-\chi \nabla \times w=\Delta v^{-}, \quad t \geq 0, x \in \mathbb{R}^{3}, \\
\operatorname{div} v^{+}=\operatorname{div} v^{-}=0, \quad t \geq 0, x \in \mathbb{R}^{3}, \\
v^{+}(x, 0)=u_{0}(x)+b_{0}(x), \quad w(x, 0)=w_{0}(x), \\
v^{-}(x, 0)=u_{0}(x)-b_{0}(x), \quad x \in \mathbb{R}^{3} .
\end{array}\right.
$$

Firstly, taking the $L^{2}\left(\mathbb{R}^{3}\right)$ inner product of $v^{+}, w$ and $v^{-}$with the above equations, respectively, and integrating by parts, we can obtain the energy estimates similar to (2.2).

Next we take the $L^{2}\left(\mathbb{R}^{3}\right)$ inner product of $\left|v^{+}\right|^{2} v^{+},|w|^{2} w$ and $\left|v^{-}\right|^{2} v^{-}$with the first three equations in (2.17), respectively, and then integrate by parts to obtain

$$
\begin{aligned}
\frac{1}{4} \frac{\mathrm{d}}{\mathrm{d} t} & \left(\left\|v^{+}(t)\right\|_{4}^{4}+\|w(t)\|_{4}^{4}+\left\|v^{-}(t)\right\|_{4}^{4}\right)+\frac{1}{2}\left(\left\|\nabla\left|v^{+}\right|^{2}\right\|_{2}^{2}+\left\|\nabla|w|^{2}\right\|_{2}^{2}+\left\|\nabla\left|v^{-}\right|^{2}\right\|_{2}^{2}\right) \\
& +\left(\left\|\left|v^{+}\right| \nabla v^{+}\right\|_{2}^{2}+\||w| \nabla w\|_{2}^{2}+\left\|\left|v^{-}\right| \nabla v^{-}\right\|_{2}^{2}\right)+2 \chi\|w\|_{4}^{4}+\||w| \operatorname{div} w\|_{2}^{2} \\
= & \int_{\mathbb{R}^{3}}\left(\pi+|b|^{2}\right)\left(v^{+} \cdot \nabla\left|v^{+}\right|^{2}+v^{-} \cdot \nabla\left|v^{-}\right|^{2}\right) \mathrm{d} x-\int_{\mathbb{R}^{3}}(\operatorname{div} w)\left(w \cdot \nabla|w|^{2}\right) \mathrm{d} x \\
& +\chi \int_{\mathbb{R}^{3}}\left|v^{+}\right|^{2} v^{+} \cdot(\nabla \times w) \mathrm{d} x+\frac{\chi}{2} \int_{\mathbb{R}^{3}}|w|^{2} w \cdot\left(\nabla \times\left(v^{+}+v^{-}\right)\right) \mathrm{d} x \\
& +\chi \int_{\mathbb{R}^{3}}\left|v^{-}\right|^{2} v^{-} \cdot(\nabla \times w) \mathrm{d} x \\
:= & I I_{1}+I I_{2}+I I_{3}+I I_{4}+I I_{5} .
\end{aligned}
$$

We now bound the above terms one by one. For $I I_{2}$, we have

$$
\left|I I_{2}\right| \leq\||w| \nabla w\|_{2}\left\|\nabla|w|^{2}\right\|_{2} \leq \frac{1}{2}\left\|\nabla|w|^{2}\right\|_{2}^{2}+\frac{1}{2}\||w| \nabla w\|_{2}^{2} .
$$

It follows from the integration by parts, we see

$$
\begin{aligned}
\left|I I_{3}\right| & =\chi\left|\int_{\mathbb{R}^{3}} w \cdot\left(\nabla \times\left|v^{+}\right|^{2} v^{+}\right) \mathrm{d} x\right| \leq C\|w\|_{4}\left\|\left|v^{+}\right| \nabla v^{+}\right\|_{2}\left\|v^{+}\right\|_{4} \\
& \leq \frac{1}{2}\left\|\left|v^{+}\right| \nabla v^{+}\right\|_{2}^{2}+C\|w\|_{4}^{4}+C\left\|v^{+}\right\|_{4}^{4} .
\end{aligned}
$$


Similarly, we have

$$
\left|I I_{4}\right| \leq \frac{1}{2}\||w| \nabla w\|_{2}^{2}+C\|w\|_{4}^{4}+C\left\|v^{+}\right\|_{4}^{4}+C\left\|v^{-}\right\|_{4}^{4}
$$

and

$$
\left|I I_{5}\right| \leq \frac{1}{2}\left\|\left|v^{-}\right| \nabla v^{-}\right\|_{2}^{2}+C\|w\|_{4}^{4}+C\left\|v^{-}\right\|_{4}^{4} .
$$

The process for estimating $I I_{1}$ is more subtle. It follows from Hölder's inequality and Lemma 2.1 that

$$
\begin{aligned}
\left|I I_{1}\right| & \leq\left\|\left(\pi+|b|^{2}\right)\right\|_{4}\left(\left\|v^{+}\right\|_{4}\left\|\nabla\left|v^{+}\right|^{2}\right\|_{2}+\left\|v^{-}\right\|_{4}\left\|\nabla\left|v^{-}\right|^{2}\right\|_{2}\right) \\
& \leq\left\|\partial_{3}\left(\pi+|b|^{2}\right)\right\|_{p}^{\frac{1}{3}}\left\|\nabla\left(\pi+|b|^{2}\right)\right\|_{\frac{8 p}{7 p-4}}^{\frac{2}{3}}\left(\left\|v^{+}\right\|_{4}\left\|\nabla\left|v^{+}\right|^{2}\right\|_{2}+\left\|v^{-}\right\|_{4}\left\|\nabla\left|v^{-}\right|^{2}\right\|_{2}\right) .
\end{aligned}
$$

To estimate the term involving $\nabla\left(\pi+|b|^{2}\right)$, we take the divergence of the first equation of (2.17) and find

$$
\pi+|b|^{2}=(-\Delta)^{-1} \nabla \cdot\left(v^{-} \cdot \nabla v^{+}\right)
$$

by $\operatorname{div} v^{+}=\operatorname{div}(\nabla \times w)=0$. Then the Calderón-Zygmund inequality, Hölder's inequality and the interpolation inequality imply that

$$
\begin{aligned}
\left\|\nabla\left(\pi+|b|^{2}\right)\right\|_{\frac{8 p}{7 p-4}} & =\left\|\nabla(-\Delta)^{-1} \nabla \cdot\left(v^{-} \cdot \nabla v^{+}\right)\right\|_{\frac{8 p}{7 p-4}} \\
& \leq\left\|v^{-} \cdot \nabla v^{+}\right\|_{\frac{8 p}{7 p-4}} \\
& \leq C\left\|\nabla v^{+}\right\|_{2}\left\|v^{-}\right\|_{\frac{8 p}{3 p-4}}=C\left\|\nabla v^{+}\right\|_{2}\left\|\left|v^{-}\right|^{2}\right\|_{\frac{4 p}{3 p-4}}^{\frac{1}{2}} \\
& \leq C\left\|\nabla v^{+}\right\|_{2}\left\|\left|v^{-}\right|^{2}\right\|_{2}^{\frac{7 p-12}{8 p}}\left\|\nabla\left|v^{-}\right|^{2}\right\|_{2}^{\frac{12-3 p}{8 p}} .
\end{aligned}
$$

Similarly, we have

$$
\left\|\nabla\left(\pi+|b|^{2}\right)\right\|_{\frac{8 p}{7 p-4}} \leq C\left\|\nabla v^{-}\right\|_{2}\left\|\left|v^{+}\right|^{2}\right\|_{2}^{\frac{7 p-12}{8 p}}\left\|\nabla\left|v^{+}\right|^{2}\right\|_{2}^{\frac{12-3 p}{8 p}} .
$$

If $p>\frac{12}{7}$, combining the above two estimates, we see

$$
\begin{aligned}
\left|I I_{1}\right| \leq & C\left\|\partial_{3}\left(\pi+|b|^{2}\right)\right\|_{p}^{\frac{1}{3}}\left\|\nabla v^{+}\right\|_{2}^{\frac{2}{3}}\left\|v^{-}\right\|_{4}^{\frac{7 p-12}{6 p}}\left\|\nabla\left|v^{-}\right|^{2}\right\|_{2}^{\frac{4-p}{4 p}}\left\|v^{+}\right\|_{4}\left\|\nabla\left|v^{+}\right|^{2}\right\|_{2} \\
& +C\left\|\partial_{3}\left(\pi+|b|^{2}\right)\right\|_{p}^{\frac{1}{3}}\left\|\nabla v^{-}\right\|_{2}^{\frac{2}{3}}\left\|v^{+}\right\|_{4}^{\frac{7 p-12}{6 p}}\left\|\nabla\left|v^{+}\right|^{2}\right\|_{2}^{\frac{4-p}{4 p}}\left\|v^{-}\right\|_{4}\left\|\nabla\left|v^{-}\right|^{2}\right\|_{2} \\
\leq & \frac{1}{4}\left\|\nabla\left|v^{+}\right|^{2}\right\|_{2}^{2}+\frac{1}{4}\left\|\nabla\left|v^{+}\right|^{2}\right\|_{2}^{2} \\
& +C\left\|\partial_{3}\left(\pi+|b|^{2}\right)\right\|_{p}^{\frac{8 p}{3(5 p-4)}}\left\|\nabla v^{+}\right\|_{2}^{\frac{16 p}{3(5 p-4)}}\left\|v^{-}\right\|_{4}^{\frac{4(7 p-12)}{3(5 p-4)}}\left\|v^{+}\right\|_{4}^{\frac{8 p}{5 p-4}} \\
& +C\left\|\partial_{3}\left(\pi+|b|^{2}\right)\right\|_{p}^{\frac{8 p}{3(5 p-4)}}\left\|\nabla v^{-}\right\|_{2}^{\frac{16 p}{3(5 p-4)}}\left\|v^{+}\right\|_{4}^{\frac{4(7 p-12)}{3(5 p-4)}}\left\|v^{-}\right\|_{4}^{\frac{8 p}{5 p-4}}
\end{aligned}
$$




$$
\begin{aligned}
\leq & \frac{1}{4}\left\|\nabla\left|v^{+}\right|^{2}\right\|_{2}^{2}+\frac{1}{4}\left\|\nabla\left|v^{+}\right|^{2}\right\|_{2}^{2} \\
& +C\left(\left\|\partial_{3}\left(\pi+|b|^{2}\right)\right\|_{p}^{\frac{8 p}{7 p-12}}+\left\|\nabla v^{+}\right\|_{2}^{2}\right)\left(\left\|v^{-}\right\|_{4}^{\frac{4(7 p-12)}{3(3 p-4)}}+\left\|v^{+}\right\|_{4}^{4}\right) \\
& +C\left(\left\|\partial_{3}\left(\pi+|b|^{2}\right)\right\|_{p}^{\frac{8 p}{7 p-12}}+\left\|\nabla v^{-}\right\|_{2}^{2}\right)\left(\left\|v^{+}\right\|_{4}^{\frac{4(7 p-12)}{3(3 p-4)}}+\left\|v^{-}\right\|_{4}^{4}\right) \\
\leq & \frac{1}{4}\left\|\nabla\left|v^{+}\right|^{2}\right\|_{2}^{2}+\frac{1}{4}\left\|\nabla\left|v^{+}\right|^{2}\right\|_{2}^{2} \\
& +C\left(\left\|\partial_{3}\left(\pi+|b|^{2}\right)\right\|_{p}^{\frac{8 p}{7 p-12}}+\left\|\nabla v^{+}\right\|_{2}^{2}+\left\|\nabla v^{-}\right\|_{2}^{2}\right)\left(1+\left\|v^{-}\right\|_{4}^{4}+\left\|v^{+}\right\|_{4}^{4}\right) .
\end{aligned}
$$

The case $p=\frac{12}{7}$ can be similarly dealt with.

Summarily, we conclude that

$$
\begin{aligned}
& \frac{1}{4} \frac{\mathrm{d}}{\mathrm{d} t}\left(\left\|v^{+}(t)\right\|_{4}^{4}+\|w(t)\|_{4}^{4}+\left\|v^{-}(t)\right\|_{4}^{4}\right) \\
& \quad \leq C\left(1+\left\|\partial_{3}\left(\pi+|b|^{2}\right)\right\|_{p}^{\frac{8 p}{7 p-12}}+\left\|\nabla v^{+}\right\|_{2}^{2}+\left\|\nabla v^{-}\right\|_{2}^{2}\right)\left(1+\left\|v^{-}\right\|_{4}^{4}+\|w\|_{4}^{4}+\left\|v^{+}\right\|_{4}^{4}\right) .
\end{aligned}
$$

Thus, Gronwall's inequality together with the assumption (1.5) and the energy estimates gives the desired $L^{4}$ estimates $(2.12)$ and thus the solution $(u, w, b)$ can be extended beyond $T$.

\section{Competing interests}

The authors declare that they have no competing interests.

\section{Authors' contributions}

ZX wrote the first draft and $\mathrm{HY}$ corrected and improved it. Both authors read and approved the final draft.

\section{Acknowledgements}

The authors would like to thank the referees for their valuable comments and remarks. This work was partially supported by the NNSF of China (No. 11101068), the Sichuan Youth Science \& Technology Foundation (No. 2011JQ0003), the SRF for ROCS, SEM, and the Fundamental Research Funds for the Central Universities (ZYGX2009X019).

\section{Received: 1 August 2012 Accepted: 12 November 2012 Published: 27 November 2012}

\section{References}

1. Ahmadi, G, Shahinpoor, M: Universal stability of magneto-micropolar fluid motions. Int. J. Eng. Sci. 12, 657-663 (1974)

2. Yuan, J: Existence theorem and blow-up criterion of the strong solutions to the magneto-micropolar fluid equations. Math. Methods Appl. Sci. 31, 1113-1130 (2008)

3. Galdi, GP, Rionero, S: A note on the existence and uniqueness of solutions of the micropolar fluid equations. Int J. Eng. Sci. 15, 105-108 (1977)

4. Ortega-Torres, EE, Rojas-Medar, MA: Magneto-micropolar fluid motion: global existence of strong solutions. Abstr. Appl. Anal. 4, 109-125 (1999)

5. Rojas-Medar, MA: Magneto-micropolar fluid motion: existence and uniqueness of strong solution. Math. Nachr. 188, 301-319 (1997)

6. Rojas-Medar, MA, Boldrini, JL: Magneto-micropolar fluid motion: existence of weak solutions. Rev. Mat. Complut. 11, 443-460 (1998)

7. Beale, JT, Kato, T, Majda, A: Remarks on the breakdown of smooth solutions for the 3-D Euler equations. Commun. Math. Phys. 94, 61-66 (1984)

8. Veiga, H: A new regularity class for the Navier-Stokes equations in $R^{n}$. Chin. Ann. Math. 16, 407-412 (1995)

9. Berselli, L, Galdi, G: Regularity criteria involving the pressure for the weak solutions of the Navier-Stokes equations. Proc. Am. Math. Soc. 130, 3585-3595 (2002)

10. Chae, D, Lee, J: Regularity criterion in terms of pressure for the Navier-Stokes equations. Nonlinear Anal. TMA 46 727-735 (2001)

11. Chen, Q, Zhang, Z: Regularity criterion via the pressure on weak solutions to the 3D Navier-Stokes equations. Proc Am. Math. Soc. 135, 1829-1837 (2007)

12. Fan, J, Jiang, $\mathrm{S}, \mathrm{Ni}$, G: On regularity criteria for the $n$-dimensional Navier-Stokes equations in terms of the pressure. J. Differ. Equ. 244, 2963-2979 (2006)

13. Penel, P, Pokorny, M: Some new regularity criteria for the Navier-Stokes equations containing gradient of the velocity. Appl. Math. 49, 483-493 (2004) 
14. Serrin, J: On the interior regularity of weak solutions of the Navier-Stokes equations. Arch. Ration. Mech. Anal. 9, 187-195 (1962)

15. Zhou, Y: Regularity criteria in terms of pressure for the 3D Navier-Stokes equations in a generic domain. Math. Ann. 328, 173-192 (2004)

16. Zhou, Y: On a regularity criterion in terms of the gradient of pressure for the Navier-Stokes equations in $\mathbb{R}^{N}$. Z. Angew. Math. Phys. 57, 384-392 (2006)

17. Cao, C, Titi, ES: Global regularity criterion for the 3D Navier-Stokes equations involving one entry of the velocity gradient tensor. Arch. Ration. Mech. Anal. 202, 919-932 (2011)

18. Zhou, Y, Pokorny, M: On the regularity of the solutions of the Navier-Stokes equations via one velocity component. Nonlinearity 23, 1097-1107 (2010)

19. Cao, C, Titi, ES: Global well-posedness of the three-dimensional viscous primitive equations of large scale ocean and atmosphere dynamics. Ann. Math. 166, 245-267 (2007)

20. Cao, C, Titi, ES: Regularity criteria for the three-dimensional Navier-Stokes equations. Indiana Univ. Math. J. 57 2643-2661 (2008)

21. Caflisch, R, Klapper, I, Steele, G: Remarks on singularities, dimension and energy dissipation for ideal hydrodynamics and MHD. Commun. Math. Phys. 184, 443-455 (1997)

22. Wu, J: Viscous and inviscid magnetohydrodynamics equations. J. Anal. Math. 73, 251-265 (1997)

23. He, C, Xin, Z: On the regularity of weak solutions to the magnetohydrodynamic equations. J. Differ. Equ. 213, 235-254 (2005)

24. He, C, Xin, Z: Partial regularity of suitable weak solutions to the incompressible magnetohydrodynamic equations. J. Funct. Anal. 227, 113-152 (2005)

25. Chen, Q, Miao, C, Zhang, Z: On the regularity criterion of weak solution for the 3D viscous magneto-hydrodynamics equations. Commun. Math. Phys. 284, 919-930 (2008)

26. $\mathrm{Wu}$, J: Regularity criteria for the generalized MHD equations. Commun. Partial Differ. Equ. 33, 285-306 (2008)

27. Cao, C, Wu, J: Two regularity criteria for the 3D MHD equations. J. Differ. Equ. 248, 2263-2274 (2010)

28. Liu, Q, Zhao, J, Cui, S: A regularity criterion for the three-dimensional nematic liquid crystal flow in terms of one directional derivative of the velocity. J. Math. Phys. 52, Article ID 033102 (2011)

29. Dong, B, Jia, Y, Chen, Z: Pressure regularity criteria of the three-dimensional micropolar fluid flows. Math. Methods Appl. Sci. 34, 595-606 (2011)

30. Dong, B, Chen, Z: Regularity criteria of weak solutions to the three-dimensional micropolar flows. J. Math. Phys. 50, Article ID 103525 (2009)

31. Yuan, B: On the regularity criteria of weak solutions to the micropolar fluid equations in Lorentz space. Proc. Am. Math. Soc. 138, 2025-2036 (2010)

32. Yuan, B: Regularity of weak solutions to magneto-micropolar fluid equations. Acta Math. Sci., Ser. B 30, 1469-1480 (2010)

33. Gala, S: Regularity criteria for the 3D magneto-micropolar fluid equations in the Morrey-Campanato space. Nonlinear Differ. Equ. Appl. 17, 181-194 (2010)

34. Geng, J, Chen, X, Gala, S: On regularity criteria for the 3D magneto-micropolar fluid equations in the critical Morrey-Campanato space. Commun. Pure Appl. Anal. 10, 583-592 (2011)

35. Wang, Y, Hu, L, Wang, Y: A Beale-Kato-Madja criterion for magneto-micropolar fluid equations with partial viscosity. Bound. Value Probl. 2011, Article ID 128614 (2011)

36. Zhang, Z, Yao, Z, Wang, X: A regularity criterion for the 3D magneto-micropolar fluid equations in Triebel-Lizorkin spaces. Nonlinear Anal. TMA 74, 2220-2225 (2011)

37. Adams, A, Fournier, JJF: Sobolev Spaces, 2nd edn. Academic Press, New York (2003)

doi:10.1186/1687-2770-2012-139

Cite this article as: Xiang and Yang: On the regularity criteria for the 3D magneto-micropolar fluids in terms of one

directional derivative. Boundary Value Problems 2012 2012:139.

\section{Submit your manuscript to a SpringerOpen ${ }^{\circ}$ journal and benefit from:}

- Convenient online submission

Rigorous peer review

- Immediate publication on acceptance

- Open access: articles freely available online

- High visibility within the field

- Retaining the copyright to your article

Submit your next manuscript at $>$ springeropen.com 